\title{
Blood equivalent phantom vs whole human blood, a comparative study
}

\author{
K. Karpienko, M. Gnyba, D. Milewska, M. S. Wróbel and M. Jędrzejewska-Szczerska* \\ Department of Metrology and Optoelectronics \\ Faculty of Electronics, Telecommunications and Informatics \\ Gdańsk University of Technology \\ 11/12 Narutowicza 80-233 Gdańsk, Poland \\ *mjedrzej@eti.pg.gda.pl
}

Received 30 July 2015

Accepted 26 October 2015

Published 22 December 2015

\begin{abstract}
Preclinical research of biomedical optoelectronic devices is often performed with the use of blood phantoms - a simplified physical model of blood. The aim of this study is the comparison and distinction between blood phantoms as well as whole human blood measurements. We show how the use of such phantoms may influence the incorrect interpretation of measured signal. On the other hand, we highlight how the use of blood phantoms enables to investigate the phenomena that otherwise are almost impossible to be noticed.
\end{abstract}

Keywords: Blood phantoms; low-coherence interferometry; absorption spectroscopy; Raman spectroscopy.

\section{Introduction}

Novel optical measurement ${ }^{1-4}$ and imaging ${ }^{5-9}$ methods for biomedicine require preclinical optimization and calibration. Such calibration is performed on standardized phantoms - simplified physical models of tissues. ${ }^{10-12}$ Especially, phantoms simulating optical properties of $\operatorname{skin}^{13-15}$ are used for testing noninvasive optical devices. Similarly, blood monitoring ${ }^{16,17}$ devices have to be calibrated with the use of blood-equivalent phantoms as well. Typically, a physical blood phantom is obtained by suspension of red blood cells (RBCs) into the isotonic saline solution. The use of phantoms allows to improve repeatability of measurement results, because phantoms are more stable over time. Moreover, using phantoms instead of human blood is much cheaper and more convenient. For these reasons, blood phantoms are commonly used in biomedical research as a simplified physical model of human blood. However, it is important to investigate how application of physical blood phantom instead of human blood, can influence on measurements results. This study presents the

*Corresponding author.

This is an Open Access article published by World Scientific Publishing Company. It is distributed under the terms of the Creative Commons Attribution 4.0 (CC-BY) License. Further distribution of this work is permitted, provided the original work is properly cited. 
comparison and differentiation between bloodequivalent phantoms and whole human blood measurements. We show how the use of such phantoms influences the measured signal and leads to its incorrect interpretation. On the other hand, we highlight how the use of blood phantoms enables us to investigate the phenomena that are otherwise obscured by other blood components, thus almost impossible to notice. We use two complementary methods: low-coherence interferometry ${ }^{18,19}$ and absorption spectroscopy ${ }^{20-24}$ to detect the differences in properties of the sample. The measurements were performed on whole blood, blood plasma as well as a blood-equivalent phantom (see Sec. 2.1.3).

\section{Materials and Methods}

\subsection{Investigated samples}

In order to detect differences between blood-equivalent phantoms and whole blood, we have measured whole human blood ${ }^{25}$ as well as each of its main constituents: red blood cells and blood plasma. Blood plasma mostly consists of water (approximately 90\%), proteins (about 7\%) and small quantities of salt and minerals. ${ }^{25,26}$

\subsubsection{Whole human blood}

Blood samples were obtained from healthy volunteers and therefore our measurement range of the haematocrit (HCT) was limited to the values of about $30 \%$ to $50 \%$. A set of $2 \mathrm{~mL}$ blood samples with various HCT levels was provided by the Gdańsk Blood Donor Center, which also performed reference measurement of the haematological parameters in each blood sample using the standard procedure. Both measurements were performed up to $6 \mathrm{~h}$ from the blood donation to ensure correct and consistent results.

\subsubsection{Blood plasma}

Blood plasma was obtained with regard to blood plasma preparation protocols by centrifugation of the whole blood sample at high speed for $15 \mathrm{~min}$. There are three distinct layers resulting from centrifugation: plasma, white blood cells and RBCs (from top to bottom) from which the plasma is selected.

\subsubsection{Blood phantom}

The blood phantom is obtained through the suspension of RBCs into the isotonic saline solution. ${ }^{11}$ The RBCs are obtained by multiple centrifugation of whole blood sample, which divides the blood into separate components, that is: plasma and RBCs. The supernatant plasma is removed from the sample and discarded. Subsequently, the remaining RBCs are washed with isotonic saline solution. Thus, plasma is completely removed and exchanged for saline.

\subsection{Experimental setup}

An experimental setup was designed and built accordingly to the layout shown in Fig. 1. It consists of a low-coherence interferometer (Fig. 1 top part of image), an optical absorption spectrometer (Fig. 1 middle part of image) and a Raman spectrometer setup (Fig. 1 bottom part of image).

\subsubsection{Low-coherence interferometry}

In low-coherence interferometer ${ }^{27,28}$ (shown in Fig. 2), we applied three interchangeable superluminescent diodes (SLED) with Gaussian spectral density (by Superlum Ltd., Ireland) type: S-930B-I-10 (central wavelength $\lambda=935 \mathrm{~nm}$, bandwidth $\left.\Delta \lambda_{\mathrm{FWHM}}=70 \mathrm{~nm}\right), \quad$ S1300-G-I-20 $\quad(\lambda=1290 \mathrm{~nm}$, $\left.\Delta \lambda_{\text {FWHM }}=50 \mathrm{~nm}\right), \quad$ and $\quad$ S-1550-G-I-20 $\quad(\lambda=$ $1550 \mathrm{~nm}, \Delta \lambda_{\text {FWHM }}=45 \mathrm{~nm}$ ) as a broadband source. An Ando AQ6319 optical spectrum analyzer with resolution bandwidth set to $1 \mathrm{~nm}$ was used in the

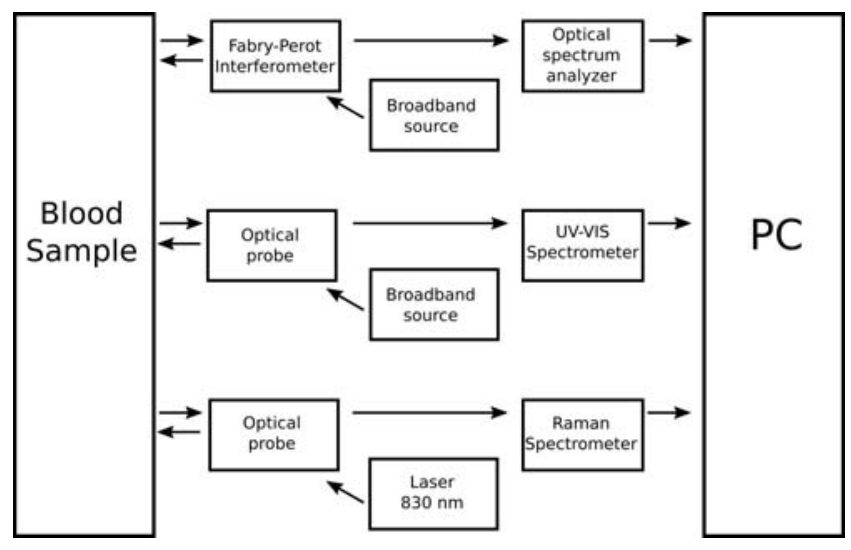

Fig. 1. The layout of experimental setup: low-coherence interferometer (top part of image), optical absorption spectrometer (middle part of image), Raman spectrometer (bottom part of image). 


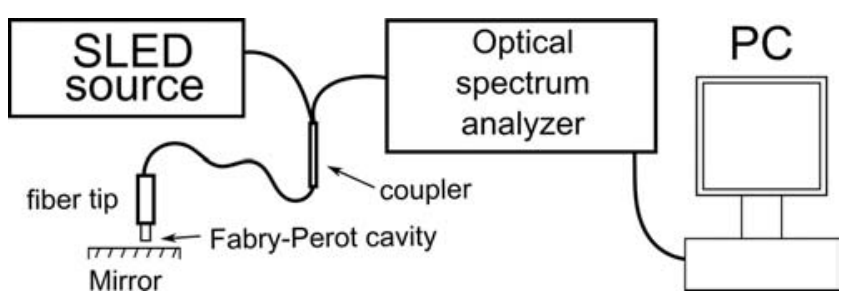

Fig. 2. Low-coherence interferometer laboratory setup.

detection setup. All interconnections, as well as the coupler, were made from single-mode commercially available, telecommunications fiber SMF-28. The Fabry-Perot interferometer was formed by the uncoated end surface of the single-mode fiber and the silver mirror. In order to precisely control the position of the optical fiber, the micromechanical custom-made system was utilized. The Fabry-Perot cavity had the length of $200 \mu \mathrm{m}$, visibility of the measured signal fringes reached the value up to 0.95 .

\subsubsection{Absorption spectroscopy}

The absorption spectra were recorded on a custom made laboratory setup in a transmission mode. The absorption spectroscopy measurement system (Fig. 3) comprises of the commercial miniature CCD-based VIS-NIR $(\sim 400-900 \mathrm{~nm})$ spectrometer (USB4000, Ocean Optics Inc., USA), a tungsten light source, delivery and collection fibers, and a sample holder with vertical geometry (Fig. 3).

Samples were introduced into a $0.1 \mathrm{~mm}$ thick rectangular quartz capillary to reduce the optical path length, thus allowing for measurements of whole, undiluted blood samples. This was crucial because very high absorbance of blood, in the region of the Soret and $Q$ bands, rendered them unusable due to noise, in the case of very high HCT\% levels. Therefore, the heavily-distorted spectra were usually truncated to the usable NIR region. The spectra were later pre-processed with common de-noising algorithms such as Savitzky-Golay and fast Fourier transform filtering. ${ }^{29}$ The spectra were also normalized with a Standard Normal Variate normalization algorithm. The integration time was $200 \mathrm{~ms}$ with over 20 averages for blood and RBC samples.

\subsubsection{Raman spectroscopy}

Raman spectroscopy setup for measurements of blood samples and related materials utilizes $830 \mathrm{~nm}$ excitation wavelength to reduce influence of fluorescence and other optical background signals. It is based on pre-commercial Raman spectrometer Ramstas developed by the VTT - Technical Research Center of Finland. Laser beam is transmitted through the fiber to the probe, filtered and focused on the sample. Power on the sample was $100 \mathrm{~mW}$, which reduces the risk of thermal damage of the sample. Collected scattered radiation is filtered to remove laser wavelength and transmitted to spectrograph and TE-cooled CCD array $(250 \mathrm{~K})$. Signals are collected in Stokes range from 200 to $2000 \mathrm{~cm}^{-1}$. Spectral resolution of the setup is about $8 \mathrm{~cm}^{-1}$. Averaging and instrument background correction were applied during spectra acquisition (time of single acquisition was 2 s., i.e., after 150 to 300 averages).

\subsection{Measurement procedure}

The subsequent experimental process was conducted carefully, all of the relevant laboratory procedures were followed, especially the procedure related to controlling the temperature of liquid samples. For each sample under investigation, the measurement signals from each setup were recorded for further analysis. Spectral characteristics of optical parameters, such as the complex refractive index, dispersion of refractive index, absorption and

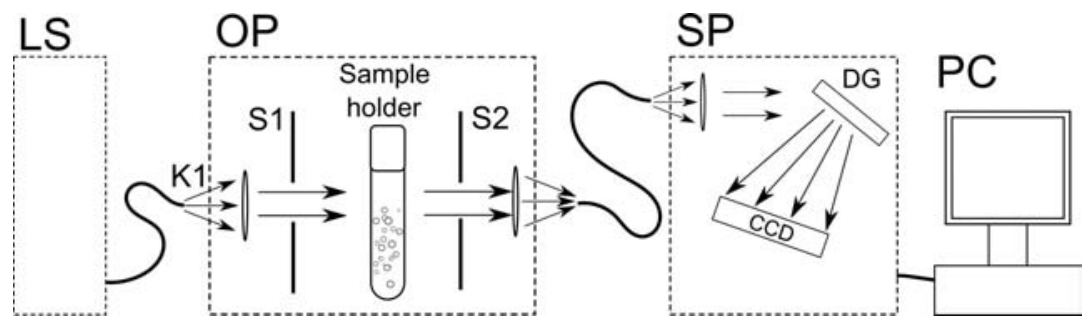

Fig. 3. Absorption spectroscopy laboratory setup (LS — broadband light source, OP — optical probe, SP — spectrometer, K1 collimator, S1, S2 - slits, DG - diffraction grating). 


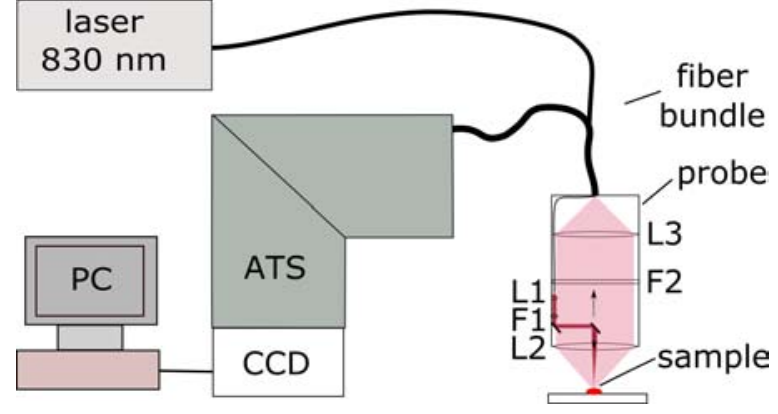

Fig. 4. Raman spectroscopy laboratory setup. L1-L3 - lenses, F1 — laser line filter, F2 - longpass filter, ATS - axial transmissive spectrograph.

Raman spectra were investigated by means of optical and Raman spectroscopy, and by low-coherence interferometric measurements carried out for multiple excitation wavelengths.

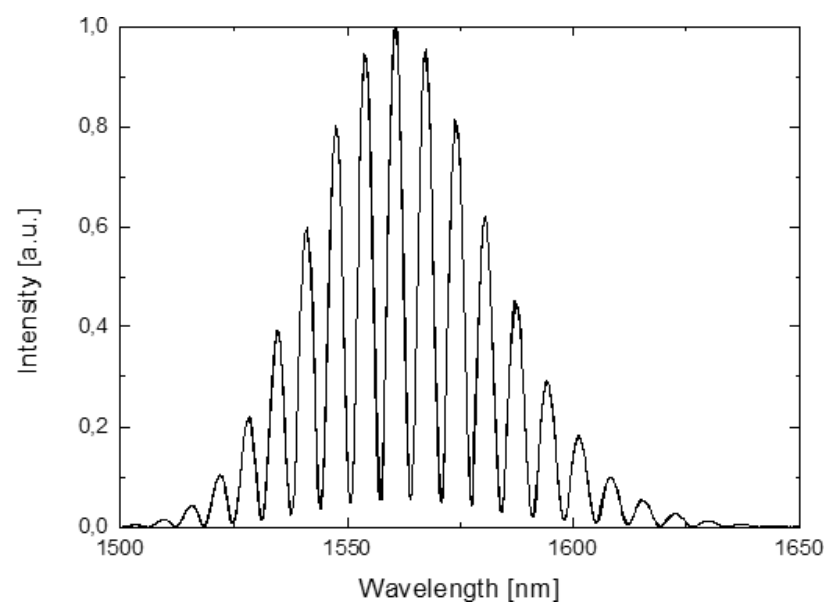

(a)

\section{Results}

\subsection{Low-coherence interferometry}

More than 100 measurements of blood plasma and saline, with the use of low-coherence interferometry setup, were conducted. Representative registered spectra for the central wavelength of $1550 \mathrm{~nm}$ are presented in Fig. 5.

Spectral separation for plasma and saline were obtained from registered spectra. Then, dispersion of refractive indices of these two substances were calculated (see Fig.6) using previously developed and validated procedures. ${ }^{30,31}$ The reference measurement (empty Fabry-Perot cavity) was used for verification of working conditions of the measurement setup.

Analysis of obtained results allowed us to confirm the unequivocal relationship between the measured

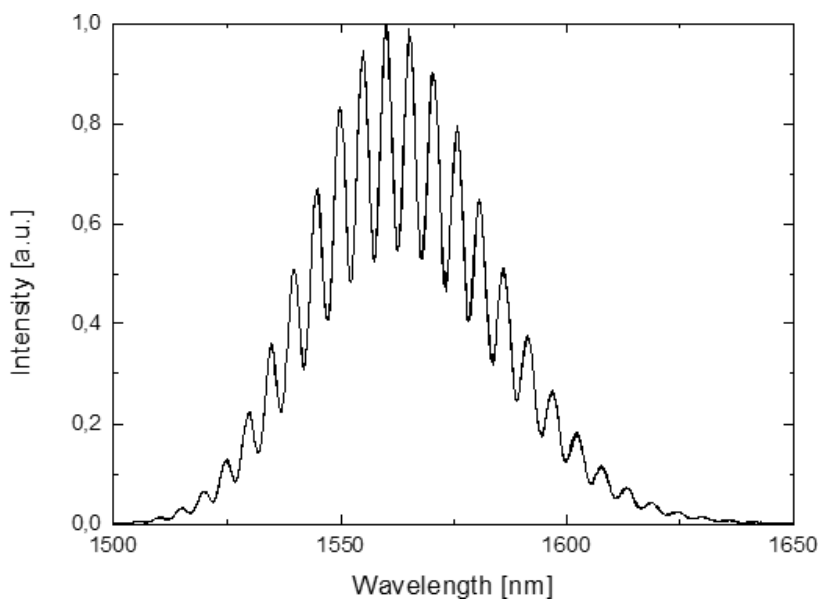

(b)

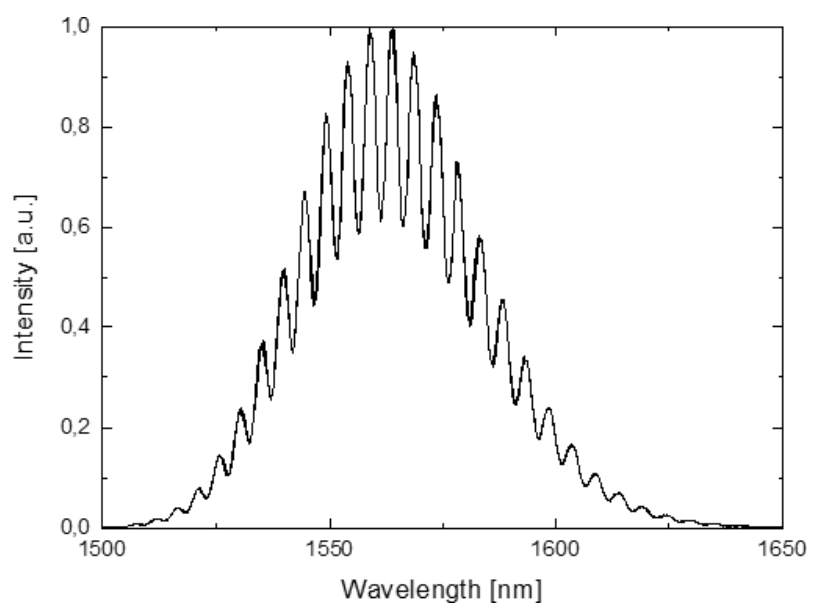

(c)

Fig. 5. Representative registered spectra for the central wavelength of $1550 \mathrm{~nm}$ : (a) reference measurement; (b) saline; (c) plasma. 


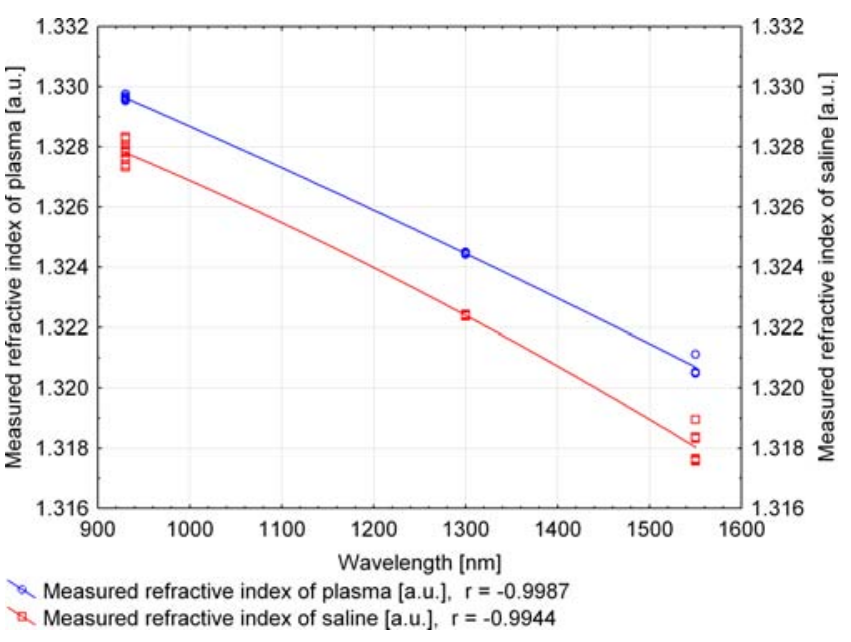

Fig. 6. Dispersion characteristics of saline and plasma.

signal from low-coherence interferometer and the refractive index of samples shown in Fig. 6.

Calculated strong negative $r$-Pearson correlations $(r=-0.9987$ for plasma and $r=-0.9944$ for saline) confirm the precision of measured refractive indices of blood plasma as well as saline solution. It can be concluded that the refractive index of plasma is greater than that of saline by about 0.002 at $930 \mathrm{~nm}$ and this difference increases with the wavelength, up to about 0.003 at $1550 \mathrm{~nm}$.

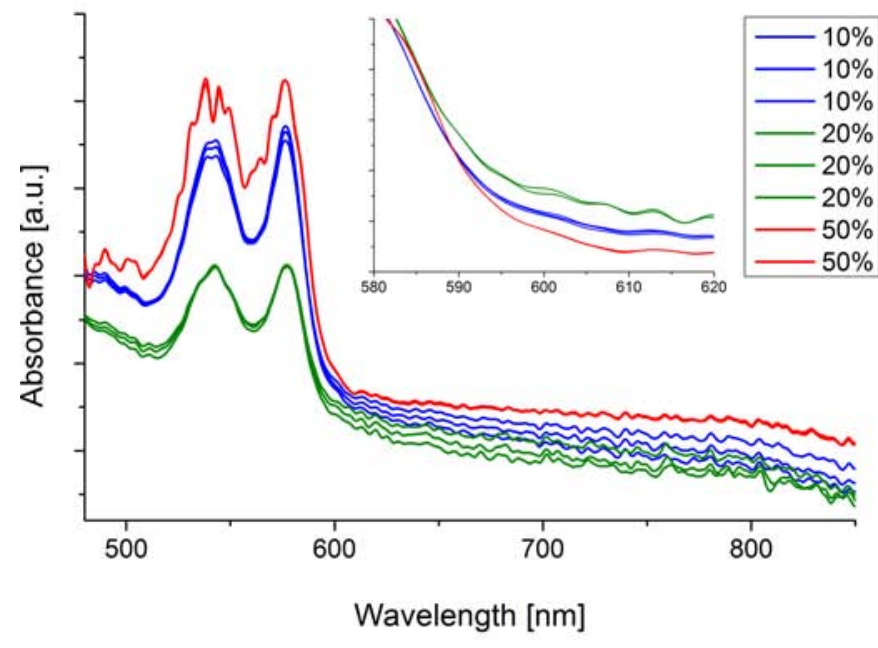

(a)

\subsection{Absorption spectroscopy}

Absorption spectra of whole human blood are dominated by characteristic haemoglobin absorption bands, the Soret band at $420 \mathrm{~nm}$ and a double Q-band in the $500-600 \mathrm{~nm}$ range. ${ }^{32,33}$ The remaining parts of the spectra are rather flat and slightly inclined. Due to high absorption causing low signal-to-noise ratio for wavelengths near Soret band, we focus our analysis on longer wavelengths. In order to perform absorption spectroscopy measurements, we have prepared a range of phantoms with various HCT levels, as presented in Fig. 6(a).

It can be seen that although the haemoglobin absorption bands remain unaffected, the baseline slope of washed erythrocytes is more inclined than that of the whole blood (Fig. 7(a)). This effect is caused by the change in the scattering of the sample which influences the overall attenuation of light. The higher scattering is caused by higher refractive index mismatch ${ }^{34}$ between the $\mathrm{RBCs}^{35-37}$ and the medium surrounding them, caused by exchange of plasma with saline, of the refractive index value 1.330 and 1.328 at $930 \mathrm{~nm}$, respectively (see Fig. 7). This effect is also increased by higher concentration of RBCs. A phantom created with $50 \%$ $\mathrm{HCT}$, the same as the blood sample, shows only slightest difference in comparison with a whole

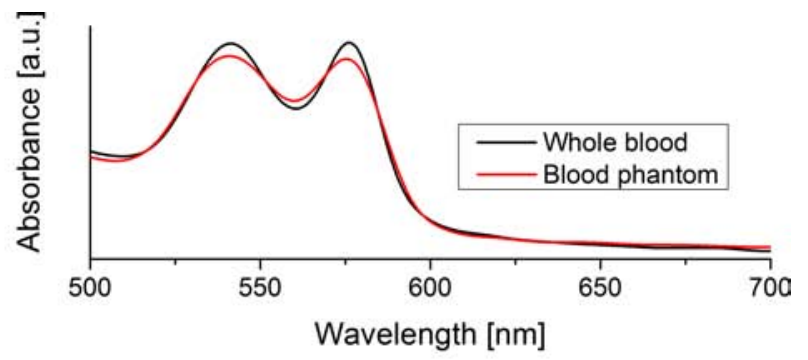

(b)

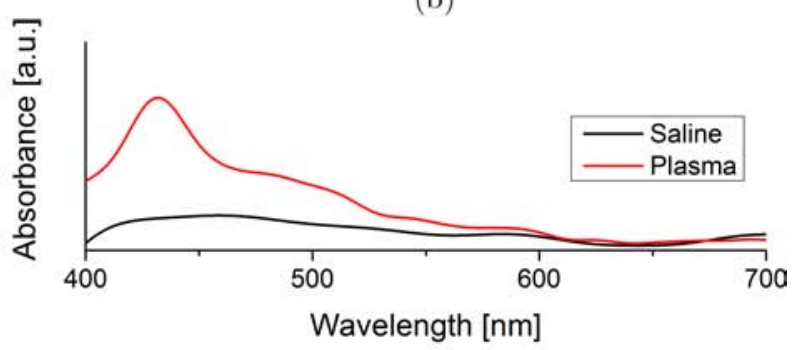

(c)

Fig. 7. Comparison of absorption spectra of blood constituents: (a) Comparison of washed erythrocytes (blood phantom) with various HCT levels; the inset presents the change in the slope of absorbance in $580-620$ nm range on normalized spectra. (b) Comparison of absorbance of whole blood and phantom at HCT of about $50 \%$ in both cases. (c) Comparison of absorbance of saline and plasma. 


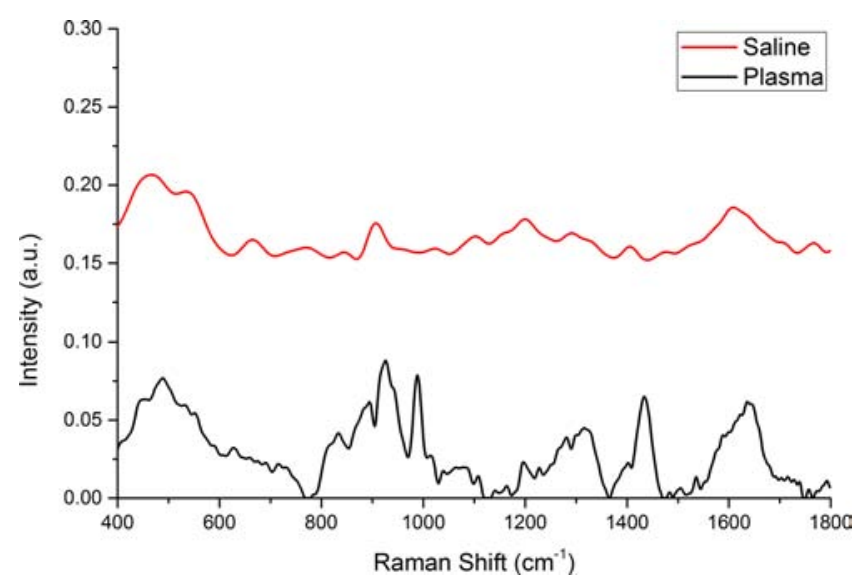

Fig. 8. Comparison of Raman spectra of blood plasma and saline.

blood sample, when considering $500-700 \mathrm{~nm}$ wavelength range. Yet, it can be seen, especially at the edges of that range, that the slope is slightly more inclined in the case of a phantom. The relation between these values is not straightforward, since the absorbance and scattering both contribute to the total absorbance of the sample, thus, they are intertwined in the case of transmission spectrometer setup. Other parameters of RBCs such as the total amount of haemoglobin, its content in erythrocyte, erythrocyte size distribution, etc. come into effect as well. On the other hand, the composition of plasma is an ensemble of a multitude of chemical substances, mainly proteins, along with platelets and white blood cells. Comparison of saline solution with plasma (Fig. 6(c)) shows that saline has no influence on the spectra, while plasma exhibits broad absorption band between 400 and $500 \mathrm{~nm}$, which corresponds to the presence of proteins and other blood cells.

\subsection{Raman spectroscopy}

We have used Raman spectroscopy to compare the chemical composition of plasma and saline. Spectrum of the plasma is strongly distorted by optical background and the number of the bands that can be clearly detected is limited. Thus, data were processed by smoothing FFT-filtering and optical background removal. ${ }^{38}$ Result of the data processing (Fig. 8) shows that the acquired spectral profile of plasma is very similar to those presented in the literature, ${ }^{39,40}$ with prominent bands related mostly to the presence of proteins. Spectrum of saline is, as expected, void of identifiable features, and the detected signal is on similar levels as the background and noise interference. Thus, we can confirm the composition of saline (water, salt) and prove that there are no detectable impurities present in the spectrum of saline.

\section{Discussion}

We used complementary optical methods: low-coherence interferometry, absorption spectroscopy, and Raman spectroscopy to investigate the differences in, both physical and chemical, composition of the samples. The measurements were performed on whole blood, blood plasma, as well as blood-equivalent phantoms prepared as a suspension of washed erythrocytes in saline. The low coherence measurements provide insight into the change of refractive index caused solely by the plasma and saline. We observed small yet important differences in refractive indices of about 0.002 at $930 \mathrm{~nm}$ that increased with the wavelength. In case of blood absorption, only a phantom with standard physiological concentration of $50 \% \mathrm{HCT}$ was comparable to the whole blood, although not precisely. However, exchange of plasma with saline causes clearly visible changes in the scattering-influenced slope in blood spectra, especially when considering smaller HCT levels, in which the volume of plasma has greater influence. Raman spectroscopy explains the difference in refractive index between plasma and saline by confirming the presence of proteins and other constituents of plasma which increase the refractive index. We have shown that blood-equivalent phantom cannot be considered as a full representation of blood. Therefore, application of such phantoms cannot be used as a calibration standard for optical biomedical devices.

The investigation covered a full range of blood parameters from healthy person as well as abnormalities in the blood composition. Therefore, we ensure that our conclusions are valid for wide range of variability in investigated samples. Presented results may find application in testing various optical detection methods in biology and medicine, such as other spectroscopic ${ }^{41-44}$ as well as low-coherence methods. ${ }^{45-47}$

In the case of not chemically-specific methods, the phantoms have certain viability as a first-trial physical model, as they are easier to handle and 
their parameters can be easily modified (ex. diluted to decrease HCT\%). However, in the case of chemically-specific methods, such as fluorescence or Raman spectroscopy, those phantoms present an over-simplified case. Most of blood sample variability is due to the changes in chemical composition of plasma, which is specific for each patient. Thus, the phantoms in which the plasma is exchanged for saline, are void of huge information (or noise) component. This in turn would cause a great reduction in sample variability, enabling to build calibration models easily, but is completely useless for a real-case application, where the individual variability is a major challenge. ${ }^{48}$

In the case of fluorescence, the blood spectra are dominated by haemoglobin fluorescence from the RBCs. The blood-equivalent phantoms should be a viable model in this case for exploring the autofluorescence and photobleaching effects, ${ }^{49}$ yet only to the extent when the fluorescence from chemical compounds in plasma can be neglected. In other cases, such phantoms are also unrealistic over-simplified models which fall short for real-application cases.

Investigation on pulse light response from absorbing and scattering materials (such as solid tissues and blood) could be a good application for discussed phantoms. This would be beneficial especially in the case of noninvasive measurement techniques, and aid in the development of fiberoptical probes and other light collection equipment. ${ }^{50}$

The use of presented blood phantoms is a widespread practice in current biophotonics research. However, recent findings show the possible solution to the problem, and propose methods which do not require previous calibration, ${ }^{51}$ thus removing the need for phantoms of any kind altogether. Yet, until such methods are fully developed, the use of phantoms still remains commonplace.

\section{Acknowledgment}

This study was partially supported by the National Science Center under the Grant No. 2011/03/D/ ST7/03540, as well as DS Programs of the Faculty of Electronics, Telecommunications and Informatics of the Gdańsk University of Technology. This study was approved by the Bioethics Committee of the Medical University of Lublin according to the
Guidelines for Good Clinical Practice on 20 December 2012 (protocol No. KE-0254/293/2012).

\section{References}

1. S. Hyttel-Sorensen et al., "Calibration of a prototype NIRS oximeter against two commercial devices on a blood-lipid phantom," Biomed. Opt. Express 4(9), 1662-1672 (2013), doi:10.1364/BOE.4.001662.

2. M. Wojdyla, S. Raj, D. Petrov, "Absorption spectroscopy of single red blood cells in the presence of mechanical deformations induced by optical traps," J. Biomed. Opt. 17(9), 97006 (2012), doi:10.1117/ 1.JBO.17.9.097006.

3. J. Chaiken et al., "Effect of hemoglobin concentration variation on the accuracy and precision of glucose analysis using tissue modulated, noninvasive, in vivo Raman spectroscopy of human blood: A small clinical study," J. Biomed. Opt. 10(3), 031111 (2005), doi:10.1117/1.1922147.

4. T. Myllylä et al., "Human heart pulse wave responses measured simultaneously at several sensor placements by two MR-compatible fibre optic methods," J. Sens. 2012, 1-8 (2012), doi:10.1155/ 2012/769613.

5. A. Vogel et al., "Using noninvasive multispectral imaging to quantitatively assess tissue vasculature," J. Biomed. Opt. 12(5), 051604 (2007), doi:10.1117/ 1.2801718 .

6. H. S. Cho et al., "High frame-rate intravascular optical frequency-domain imaging in vivo," Biomed. Opt. Express 5(1), 223-232 (2013), doi:10.1364/ BOE.5.000223.

7. T. A. Valdez et al., "Multi-color reflectance imaging of middle ear pathology in vivo," Anal. Bioanal. Chem. 407(12), 3277-3283 (2015), doi:10.1007/ s00216-015-8580-y.

8. R. L. Barbour et al., Validation of near infrared spectroscopic (NIRS) imaging using programmable phantoms, Proc. SPIE 6870, R. J. Nordstrom, Ed., p. 687002 (2008), doi:10.1117/12.769160.

9. M. Ali Ansari, S. Alikhani, E. Mohajerani, "A hybrid imaging method based on diffuse optical tomography and optomechanical method to detect a tumor in the biological phantom," Opt. Commun. 342, 12-19 (2015), doi:10.1016/j.optcom.2014. 12.035 .

10. M. S. Wróbel et al., "Multi-layered tissue head phantoms for noninvasive optical diagnostics," J. Innov. Opt. Health Sci. 8, 1541005 (2015), doi:10.1142/S1793545815410059.

11. A. V. Bykov et al., Skin phantoms with realistic vessel structure for OCT measurements, Proc. SPIE 7376, p. 73760F (2010), doi:10.1117/12.872000. 
12. B. W. Pogue, M. S. Patterson, "Review of tissue simulating phantoms for optical spectroscopy, imaging and dosimetry," J. Biomed. Opt. 11(4), 041102 (2006), doi:10.1117/1.2335429.

13. M. S. Wróbel et al., "Measurements of fundamental properties of homogeneous tissue phantoms," J. Biomed. Opt. 20(4), 045004 (2015), doi:10.1117/1. JBO.20.4.045004.

14. T. Lister, P. A. Wright, P. H. Chappell, "Optical properties of human skin," J. Biomed. Opt. 17(9), 0909011 (2012).

15. R. B. Saager et al., Multilayer silicone phantoms for the evaluation of quantitative optical techniques in skin imaging, 11 February 2010, p. 756706, doi:10.1117/12.842249.

16. I. Barman et al., "Turbidity-corrected raman spectroscopy for blood analyte detection," Anal. Chem. 81(11), 4233-4240 (2009), doi:10.1021/ ac8025509.

17. M. Meinke et al., "Chemometric determination of blood parameters using visible-near-infrared spectra," Appl. Spectrosc. 59(6), 826-835 (2005).

18. M. Jedrzejewska-Szczerska, "Measurement of complex refractive index of human blood by low-coherence interferometry," Eur. Phys. J. Spec. Top. 222(9), 2367-2372 (2013), doi:10.1140/epjst/e201302018-7.

19. K. Karpienko, M. S. Wróbel, M. JędrzejewskaSzczerska, "Determination of refractive index dispersion using fiber-optic low-coherence Fabry-Perot interferometer: Implementation and validation," Opt. Eng. 53(7), 077103 (2014), doi:10.1117/1. OE.53.7.077103.

20. S. L. Upstone, Ultraviolet/visible light absorption spectrophotometry in clinical chemistry, Encyclopedia of Analytical Chemistry, John Wiley \& Sons, New York (2006).

21. M. F. Merrick, H. L. Pardue, "Evaluation of absorption and first-and second-derivative spectra for simultaneous quantification of bilirubin and hemoglobin," Clin. Chem. 32(4), 598-602 (1986).

22. W. G. Zijlstra, A. Buursma, "Spectrophotometry of hemoglobin: Absorption spectra of bovine oxyhemoglobin, deoxyhemoglobin, carboxyhemoglobin, and methemoglobin," Comp. Biochem. Physiol. B Biochem. Mol. Biol. 118(4), 743-749 (1997).

23. V. V. Tuchin, Optical Clearing of Tissues and Blood, SPIE Press, USA (2006).

24. M. Jędrzejewska-Szczerska et al., "Spectroscopic wireless sensor of hematocrit level," Sens. Actuators Phys. 202, 8-12 (2013), doi:10.1016/j. sna.2013.03.040.

25. B. Ciesla, Hematology in Practice, F. A. Davis, Philadelphia (2007).
26. H. Theml et al., Color Atlas of Hematology: Practical Microscopic and Clinical Diagnosis, Thieme, Stuttgart; New York (2004).

27. M. Jedrzejewska-Szczerska, M. Gnyba, B. B. Kosmowski, "Low-coherence fibre-optic interferometric sensors," Acta Phys. Pol. A 120, 621-624 (2011).

28. J. Pluciski et al., "Optical low-coherence interferometry for selected technical applications," Bull. Pol. Acad. Sci. Tech. Sci. 56, 155-172 (2008).

29. R. Z. Morawski, "Measurement data processing in spectrophotometric analysers of food," Metrol. Meas. Syst. 19(4), 623-652 (2012), doi:10.2478/ v10178-012-0056-1.

30. M. Jędrzejewska-Szczerska, "Response of a new lowcoherence fabry-perot sensor to hematocrit levels in human blood," Sensors 14(4), 6965-6976 (2014), doi:10.3390/s140406965.

31. M. Jędrzejewska-Szczerska, M. Gnyba, M. Kruczkowski, Low-coherence method of hematocrit measurement, 2011 Federated Conf. Computer Science and Information Systems (FedCSIS), pp. 387-391 (2011).

32. D. J. Faber et al., "Oxygen saturation-dependent absorption and scattering of blood," Phys. Rev. Lett. 93(2), $028102 \quad$ (2004), doi:10.1103/ PhysRevLett.93.028102.

33. N. Bosschaart et al., "A literature review and novel theoretical approach on the optical properties of whole blood," Lasers Med. Sci. 29(2), 453-479 (2014), doi:10.1007/s10103-013-1446-7.

34. M. Friebel et al., "Determination of optical properties of human blood in the spectral range 250 to $1100 \mathrm{~nm}$ using Monte Carlo simulations with hematocrit-dependent effective scattering phase functions," J. Biomed. Opt. 11(3), 034021 (2006), doi:10.1117/1.2203659.

35. O. Sydoruk et al., "Refractive index of solutions of human hemoglobin from the near-infrared to the ultraviolet range: Kramers-Kronig analysis," J. Biomed. Opt. 17 (11), 115002 (2012), doi:10.1117/1. JBO.17.11.115002.

36. O. Zhernovaya et al., "The refractive index of human hemoglobin in the visible range," Phys. Med. Biol. 56(13), 4013-4021 (2011), doi:10.1088/00319155/56/13/017.

37. M. Friebel, M. Meinke, "Model function to calculate the refractive index of native hemoglobin in the wavelength range of 250-1100 nm dependent on concentration," Appl. Opt. 45(12), 2838-2842 (2006).

38. B. D. Beier, A. J. Berger, "Method for automated background subtraction from Raman spectra containing known contaminants," Analyst 134(6), 1198-1202 (2009). 
39. J. L. Pichardo-Molina et al., "Raman spectroscopy and multivariate analysis of serum samples from breast cancer patients," Lasers Med. Sci. 22(4), 229-236 (2007).

40. A. Bonifacio et al., Surface-enhanced Raman spectroscopy of blood plasma and serum using $\mathrm{Ag}$ and Au nanoparticles: A systematic study," Anal. Bioanal. Chem. 406(9/10), 2355-2365, (2014).

41. B. S. S. Anand, N. Sujatha, "Fluorescence quenching effects of hemoglobin on simulated tissue phantoms in the UV-Vis range," Meas. Sci. Technol. 23(2), 025502 (2012), doi:10.1088/0957-0233/23/2/ 025502 .

42. V. O. Korhonen et al., "Light propagation in NIR spectroscopy of the human brain," IEEE J. Sel. Top. Quantum Electron. 20(2), 1-10 (2014), doi:10.1109/JSTQE.2013.2279313.

43. D. Milej et al., "Advantages of fluorescence over diffuse reflectance measurements tested in phantom experiments with dynamic inflow of ICG," OptoElectron. Rev. 18(2), 208-213 (2010), doi:10.2478/ s11772-010-0013-z.

44. T. A. Valdez et al., "Multiwavelength fluorescence otoscope for video-rate chemical imaging of middle ear pathology," Anal. Chem. 86(20), 10454-10460 (2014), doi:10.1021/ac5030232.

45. E. Alarousu et al., Noninvasive glucose sensing in scattering media using OCT, PAS, and TOF techniques, Proc. SPIE 5474, V. V. Tuchin, Ed., pp. 33-41 (2004), doi:10.1117/12.578321.

46. A. V. Bykov et al., "Multilayer tissue phantoms with embedded capillary system for OCT and DOCT imaging," Proc. SPIE 8091, p. 80911R (2011), doi:10.1117/12.889923.

47. N. Bosschaart et al., "In vivo low-coherence spectroscopic measurements of local hemoglobin absorption spectra in human skin," J. Biomed. Opt. 16(10), 100504 (2011), doi:10.1117/1.3644497.

48. R. Pandey et al., "Emerging trends in optical sensing of glycemic markers for diabetes monitoring," TrAC Trends Anal. Chem. 64, 100-108 (2015), doi:10.1016/j.trac.2014.09.005.

49. I. Barman et al., "Effect of photobleaching on calibration model development in biological Raman spectroscopy," J. Biomed. Opt. 16(1), 011004 (2011), doi:10.1117/1.3520131.

50. C.-R. Kong et al., "A novel non-imaging optics based Raman spectroscopy device for transdermal blood analyte measurement," AIP Adv. 1, 032175 (2011), doi:10.1063/1.3646524.

51. N. Spegazzini et al., "Spectroscopic approach for dynamic bioanalyte tracking with minimal concentration information," Sci. Rep. 4, 7013, (2014), doi:10.1038/srep07013. 